\title{
An Unusual Presentation of Bilateral Internal Jugular Venous Thrombosis: A Case Report
}

\author{
Felix Uduma Uduma (Corresponding author) \\ Department of Radiology, Abia State University Teaching Hospital \\ Aba, Nigeria \\ Tel: 234-803-745-0099Ｅ-mail: felixuduma@yahoo.com \\ Yarouda M. \\ Department of Otorhinolaryngology \\ Polyclinic Bonanjo SA, Douala, Cameroon \\ Muna Wali \\ Department of Medicine, University of Yaounde, Cameroon \\ E-mail: walimuna@hotmail.com
}

Received: August 2, 2010 Accepted: August 17, 2010 doi:10.5539/gjhs.v3n1p237

\begin{abstract}
Lemierre's syndrome has been termed 'forgotten' by the medical community with the advent of antibiotics. Similarly, diffuse mediastinitis is an uncommon entity. We present a case of unusual presentation of bilateral internal jugular venous thrombosis in a 37year old ambulant male Cameroonian. This co-existed with oro/retro-pharyngeal infections and mastoiditis (shown by neck MRI) as well as diffuse mediastinitis (shown by chest CT ) Physicians should have high index of suspicion of Lemierres's syndrome, Diffuse mediastinitis, Mediastinal fibrosis, Mediastinal mass or Multifocal idiopathic fibrosclerosis as the possible differential diagnoses of bilateral internal jugular venous thrombosis.
\end{abstract}

Keywords: Thrombosis, Lemierre's syndrome, MRI, Mediastinitis

\section{Introduction}

Lemierre's syndrome (LS) is a rare disease of thrombosis of the internal jugular vein (IJV) and oro-pharyngeal infection [Baig et al, 2006, $5\{1\}$ ].

The IJV arises from the cranium at the conclusion of the sigmoid sinus and exits from the cranium through the jugular foramen. It then courses in the anterior neck lateral to the carotid artery, concluding by joining the subclavian vein to form the Brachiocephalic vein [Muller \& Darcey, 1987, pp505-8, Towbin \& Kand, 2004, pp1433-4]

IJV drains both superficial/ venous systems of the brain and upper aero-digestive tracts [Jones \& Fagan, 2007, $5(2)]$

Thrombosis of the IJV (IJVT) is rare though under-diagnosed [Muller\& Darcey, 1987, pp505-8]. With advent of antibiotics, oro-pharyngeal infections, hence Lemiere's syndrome dropped, making the central venous catheterization the commonest cause of IJVT [Muller\& Darcey, 1987, pp505-8]. The implicating organism in LS is usually the normal oral flora, anaerobe Fuso-bacterium necrophorum [Baig et al, 2006, 5\{1\}].

The neck is partition into potential spaces like pre-vertebral, retropharyngeal, peri-vascular and para-pharyngeal spaces that can serve as a portal of entry into mediastinium [Marty-Ane et al, 1999, 212-7].

\section{Case Study}

T.J, a 37year old male Cameroonian, walked into Polyclinic Bonanjo with a referral for neck MRI. Patient noticed facial and painful neck swelling a week prior to presentation without respiratory distress, fever or upper limb swelling. Neck MRI done with 0.3Tesla AIRIS machine showed asymmetrical enlargement of the proximal 
two-third of both IJV. Transverse diameter of right and left IJV as shown in the coronal section are $19.8 \mathrm{~mm}$ and $15.1 \mathrm{~mm}$ respectively compared to $5.5 \mathrm{~mm}$ of common carotid arteries. While the entire luminal signal void of the right IJV is replaced by bright $\mathrm{T} 1 \mathrm{~W}$ and $\mathrm{T} 2 \mathrm{~W}$ signals, same is seen only in anterior part of left IJV confirming patency of the posterior part [ Fig 1-Axial T1WI (TR 400, TE 15)]. This hyperintense thrombus in the right IJV has inferior curvilinear border above right IJV termination leaving the subclavian, brachiocephalic veins and superior vena cava free of thrombus (Fig 2-Coronal T1WI). Axial T2W and FLAIR sequences show peri-jugular hyperintense collection extending to the cervical inter-muscular fascial planes and mastoids (bilateral mastoiditis). Sagital enhanced T1WI shows enhancing thick oropharyngeal wall and heterogeneous retro-pharyngeal space (C2-C7 vertebrae) (Fig 3 \& Fig 4).

A diagnosis of bilateral internal jugular venous thrombosis with oro/retro-pharyngeal infections (Lemierre's syndrome) was made. Duplex Doppler ultrasonography (USS) of the neck confirmed bilateral IJV enlargement and unresponsiveness to valsava manoeuvres (Fig 5 \& 6). Both IJV harbour mobile echogenic debris (worse on the right) (Fig 6) with turbulent flow. Abdomino-pelvic ultrasonography was not contributory. Chest computed tomogram $(\mathrm{CT})$ further requested showed superior mediastinal widening with encasement of entire mediastinum by irregular diffuse thin soft tissue shadow with $\mathrm{CT}$ attenuation value of $25 \mathrm{HU}$ with associated mild right pleural effusion. (Fig 7).

Our Patient refused laboratory investigations, in lieu opted for overseas treatment and was lost to follow-up. Informed consent for publication of this case was obtained from the patient.

\section{Discussion}

Lemierre in 1936 described the first case series of septic thrombophlebitis of the IJV often complicated by systemic infections [Baig et al, 2006, $5\{1\}$ Muller \& Darcey, 1987, pp505-8, Jones \& Fagan, 2007, 5(2)]. Before the antibiotic era, Lemierre's syndrome (LS) resulted in 90\% mortality [Baig et al, 2006, 5\{1\}, Marty-Ane et al, 1999, 212-7]. With the introduction of antibiotics, the recent literature review revealed 116 case reports of Fusobacterium necrophorum, though this occurrence is likely under-reported. [Jaremko et al, 2003, 169(8)

Acute mediastinitis mainly results from oesophageal perforation following cardiac procedures [Marty-Ane et al, 1999, 212-7]. Minor causes are oropharyngeal, head and neck infections. The potentially virulent form of mediastinal infection is described as Descending Necrotising Mediastinitis (DNM). DNM in post-antibiotic era is an uncommon entity in which Corsten detected only 69 cases from 1960-1995 [Marty-Ane et al, 1999, 212-7]

The pathophysiology of internal jugular venous thrombosis (IJVT) in general is based on Virchow triad of blood vessel trauma, stasis and hypercoagulablility. IJVT aetiologies therefore include central venous catheterization, IV drug abusers, malignancies, neck massage/ trauma and head / neck surgeries. Others are hypercoagulabilities like polycythaemia, hyper-homocystinaemia, deficiencies of (Protein $\mathrm{C}$ and $\mathrm{S}$, Factor $\mathrm{V}$ Lieden, and Anti-thrombin III ); Anti-phospholipid antibody syndrome, Pregnancy, Ovarian hyper-stimulation syndrome, Anaemia, Thrombocytosis and Drugs (steroids, oral contraceptive) [Muller \& Darcey, 1987, pp505-8, Towbin \& Kand, 2004, pp1433-4, Sabire et al, 2003, 477-89, Jones \& Fagan, 2007, 5(2) Marty-Ane et al, 1999, 212-7]. Jaremko et al, 2003, 169(8), Balaen et al, 2001, pp510-12, Horstkamp et al, 1996, p280-2, Chow et al, 2000, pp1420-1425, Jaffe \& Goldhabe, 2002, pp1874-80, Hubsch et al, 1988, pp629-636, Abulafa et al, 1995, pp140-2, Caplan et al, 2008, pp777-8]

In LS the organism is usually the fusobacterial species with F. Necrophorum being the most virululent. Others are Bacteroids, Peptostreptococcus sp, Eikenelle sp, antibiotic resistant Staphylococcus aereus and other $\beta$-lactamase resistance gram positives. Baig et al, 2006, 5(1) in blood culture isolated F. Necrophorum in $81.7 \%$ of LS. In our index patient, though blood culture was not done to incriminate F.necrophorum, our diagnosis of LS was based on imaging. Besides, Jaremko et al, 2003, 169(8), stated that F.necrophorum should be considered in any patient with tenderness or swollen neck in association with pharyngitis especially in an immune-competent healthy patient. The source of this infection is from pharyngitis, tonsillitis, and mastoiditis. 2 out of 3 steps of specific pathphysiology for F necrophorum infection were seen in our patient. First step is pharyngitis and the second is invasion of lateral pharyngeal wall with septic thrombophlebitis of the IJV. But his chest CT shows small irregular mediastinal diffusely encasing soft tissue mass lesion with $\mathrm{CT}$ attenuation value of $25 \mathrm{HU}$. The impression of diffuse mediastinitis is based on Ane et al assertion that CT attenuation value of $>25 \mathrm{HU}$ is Diffuse mediastinitis and if $<20 \mathrm{HU}$ suggests mediastinal abscess [Marty-Ane et al, 1999, 212-7]. This mediastinitis we believe is the retropharyngeal infection that has spread along cervical fascial plane through neck potential anatomical spaces into the mediastinum. This is aided by gravity, breathing and negative intra-thoracic pressure [Marty-Ane et al, 1999, 212-7].

The important differential diagnoses considered in our patient are Lemierre's syndrome with diffuse mediastinitis, Mediastinal fibrosis, Mediastinal mass, Mediastinal abscess, Multifocal idiopathic 
fibrosclerosis(MIFS). Could it be the diffuse mediastinal soft tissue infilterate seen on the chest CT is the less common type of mediastinal fibrosis? But the absence of stenosis and thrombosis of mediastinal vessels like aorta, superior vena cava make it unlikely. MIFS was also less considered with absence of retro-peritoneal mass on abdomino-pelvic ultrasonography. MIFS is a fibrotic condition made up of idiopathic mediastinal fibrosis, idiopathic retroperitoneal fibrosis (Ormond's disease), Riedel Thyroiditis, Sclerosing cholangitis, Dupuytren's contraction, Keloids formation, Pulmonary fibrosis, Christian Weber syndrome [Bahjer et al, 2008, pp777-8]. Absence of a discrete focal mass(es) on chest CT rules out mediastinal mass. Thus infective aetiology is most plausible. But the puzzle is despite the constellations of head, neck and mediastinal lesions; our Patient was ambulatory and non-toxic. Could it be the usual African resilience to infections?

The commonest metastatic complication of LS is pulmonary embolism constituting 79.8\% [Sabire et al, 2005 pp477-89]. Others are cerebral sino-venous thrombosis (CSVT), pseudo-tumour cerebri, seizures, cognitive anomaly, stroke and ataxia [Sabire et al, 2005 pp477-89]. Invasion of the carotid sheaths lead to carotid artery rupture, Horner's syndrome (sympathetic chain), laryngeal oedema, trapezius muscle paralysis (compromise of spinal accessory nerve), dysphagia, brachial plexopathy and lymphoedema (thoracic duct obstruction) [Ramakantan \& Shah, 1990, pp61-3].

Though the signs and symptoms are subtle and can be overlooked, the symptomatology are varied. ]Muller and Dacey, 1987, 505-8 cited Tori et al work on IJVT where $72 \%$ of the patients had mass or neck swelling (as seen in our patient). Other features are fever, leukocytosis, neck pain, cord sign (palpable cord beneath the stenocleidomastoid), superior vena cava syndrome, jugular foramen syndrome, chylothorax, headache, and odynophagia [Caplan et al, 2008, pp777-8].

Modern imaging of choice of IJVT are MRI, magnetic resonance venography (MRV) and Duplex Doppler ultrasonography (USS). USS is bedside diagnosis that is sensitive to flow changes showing IJVT as dilated, incompressible IJV with intra-luminal echogenic clots unresponsive to Valsalva manoeuvre [Baig et al, 2006, $5\{1\}$ Muller \& Darcey, 1987, 505-8, Hubsch et al, 1988, pp629-636, Abulafa et al, 1995]. But USS does not image beneath clavicle and misses a fresh thrombus with low echogenicity. Enhanced CT makes up for these snags with better demonstration of the IJV anatomy and detection of abscess and malignancy. CT shows IJVT as a low density intra-luminal thrombus with sharply defined bright vessel wall due to uptake of contrast by the vasa vasorum [Baig et al, 2006, 5\{1\} Muller \& Darcey, 1987, 1987, 505-8]. MRI is sensitive to blood flow and detects complications. Diffusion weighted MRI detect cerebral venous infarct while perfusion weighted MRI detects venous congestion in CSVT [Sabire et al, 2005, 477-89]. MRI accurately correlates well with contrast venography to detect central thoracic venous clots in the subclavian, brachiocephalic and superior vena caval veins and venous collateralizations. Contrast venography confirms diagnosis and characterise anatomy but can worsen pre-existing thrombosis. $18^{\mathrm{F}}$-Fluoro-2-Deoxyglucose (18F-FDG) PET studies detects inflammatory or infected thrombus as the inflammatory cells are FDG- avid with increased glucose uptake [Kikuchi et al, 2004, pp888-90].

Treatment of LS is antibiotic and anticoagulations with parenteral un-fractionated Heparin. Anticoagulation is continued for 3-6 months with oral warfarin [Caplan et al, 2008, pp777-8]. Anticoagulation maintains patency of venous collaterals and reduces thrombus propagation especially if clot does not resolve completely [Chow et al, 2000. pp1420-25]. In such cases, catheter directed thrombolysis using recombinant tissue plasminogen (rTPA) is done. Other methods of treatment are percutaneous mechanical and surgical thromboectomy. Contraindications to thrombotic therapy are gastro-intestinal bleeding, hypersensitivity to thrombolytic agents, haemorrhagic stroke and surgery within the preceding 10days. If mediastinal lesion persists, surgical approach into mediastinum is achieved through cervical or thoracic route

\section{Conclusion}

Lemierre's Syndrome is a rare disease which can be diagnosed by radiologists using modern imaging tools even without any clinician suspicion or blood culture.

\section{References}

Abulafa, O., Shere, D.M., \& DeEulis, T.G. (1995). Ultrasonographic diagnosis of catheter induced combined subclavian and internal jugular vein thrombosis. American Journal of critical care, 4 (2), 140-142.

Baig, M. A., Rasheed, J., \& Subkowitz, J. V. (2006). A review of Lemierre's syndrome. The Internet of Journal of Infectious Disease, 5 (1).

Bahler, C., Hammoud, Z.T., \& Sundaram CP. (2008). Medistinal fibrosis in a patient with Idiopathic retro-peritoneal fibrosis. Interactive cardiovascular and Thoracic Surgery, 7, 336. 
Balaen, B., Geericks, K., Verganiya, P., \& Thys, K. (2001). Venous thrombosis after ovarian stimulation, Case report. Human Reproduction, 16 (3), 510-512.

Caplan, J. M., Khalpey, Z. \& Gates, J. (2008). Closed traumatic head injury; dural sinus and thrombosis. Emergency Medicine Journal, 25, 777-778.

Chow, K., Gobin, Y., Saver, J.Kidwell, C.,Dong, P., Vinuela, F. (2000). Case report of endovascular treatment of dural sinus thrombosis with rheolytic thromboectomy and intra-arterial thrombolysis. Stroke, 31, 1420-1425.

Estrera, A. S., Lanay, M.J., Grisham JM Sinn, D. P., \& Platt, M. R. (1983). Descending necrotizing mediastinitis. Surg. Gynaecol Obstet, 157, 545-552.

Gadhingkjkar, S.V., Shyamkri-Shnan, K.G., Sreedler, R., \& Sonual Kar, H. (2003). Delayed thrombosis of left internal jugular vein and left subclavian vein complication related to drainage of left subclavian vein. Intensive cardiovascular and thoracic surgery, 2, 607-610.

Horstkamp. B., Luibke, M., Kenetenich, H. Reiss, H., Buscher, U., \& Lichtenegger, W. (1996). Internal iugular vein thrombosis caused by resistance to activated protein $\mathrm{C}$ as a complication of ovarian stimulation after in vitro fertilization. Human Reproduction, 2 (2), 280-282.

Hubsch, R. L., Stinglbower, B.W., \& Schwaighafer, F.M, et al. (1988). Internal Jugular and subclavian vein thrombosis caused by central venous catheters. Evaluation using Doppler blood flow Imaging. Journal of Ultrasound in Medicine, 17 (11), 629-636.

Jaffe, H. V., \& Goldhabe, S. Z. (2002). Current perspective of upper extremity deep vein thrombosis. Circulation, 106, 1874-1880.

Jaremko, J. L., Kirton, A., \& Brenner, J. L. (2003). A 12 year old girl with pharyngitis, meningitis, sino-venous thrombosis, CMAJ, 169 (8).

Jones, N. E.., \& Fagan, J. I. (2007). Internal jugular veins thrombosis; Case study and Review of Literaure. The Internet Journal of Otorrhinology, 6(2) ISSN 1528-8420.

Kikuchi, M., Yamamoto E., Shiomi Y., Nakamoto, Y., Shiomi, Y., Fujiwara, K., \& et al. (2004). Internal venous thrombosis with marked accumulation of FDG. British Journal of Radiology 77, 888-890.

Marty-Ane, C. M. Berthet, P., Alric, P., Pegis, J D., Rouviere, P \& Mary, H., et al. (1999). Management of descending necrotising mediastinitis: an aggressive treatment for an aggressive disease. Ann Thoracic Surg, 68, 212-217.

Muller, D. K., \& Dacey, M. J. (1987). Internal jugular vein thrombosis. Radiology, 102 (2), 505-8.

Ramakantan, R., \&. Shah, P. (1990). Dysphagia due to mediastinal fibrosis in advanced pulmonary Tuberculosis. American Journal of Roentgenology, 154, 61-63.

Sabire, G., Tabarki, B. \& Saunders, D. E. (2005). Cerebral venous sinus thrombosis in children, risk factors, presrntation, diagnosis and outcome. Brain, 128 (3), 477-489.

Towbin, A. J., \& Kand, E. (2004). A review of 2 cases of fenestrated Internal jugular veins as seen by CT Angiography. American Journal of Neuro-radiology, 25, 1433-1434.

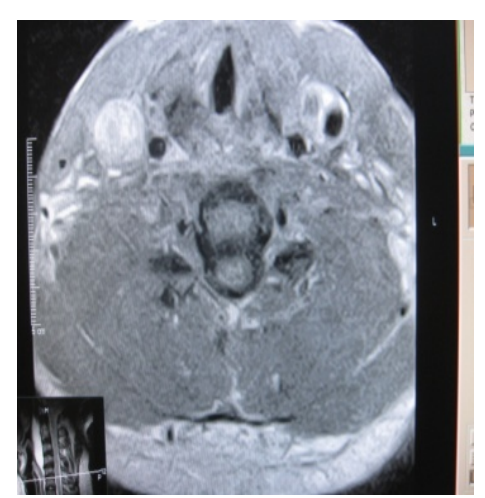

Figure 1.

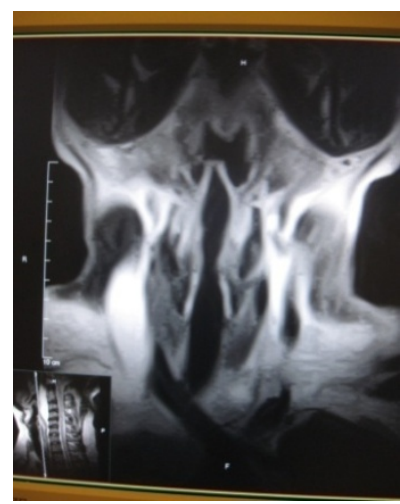

Figure 2. 


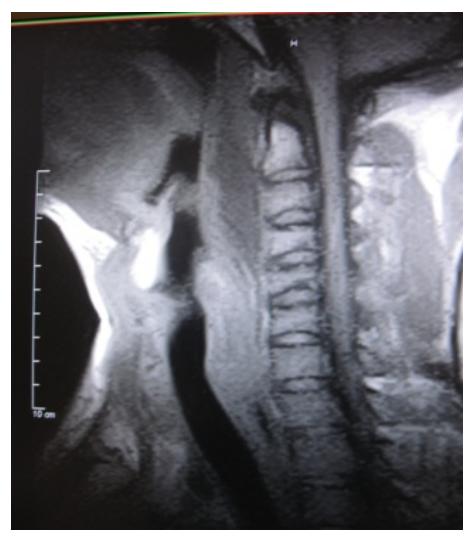

Figure 3.

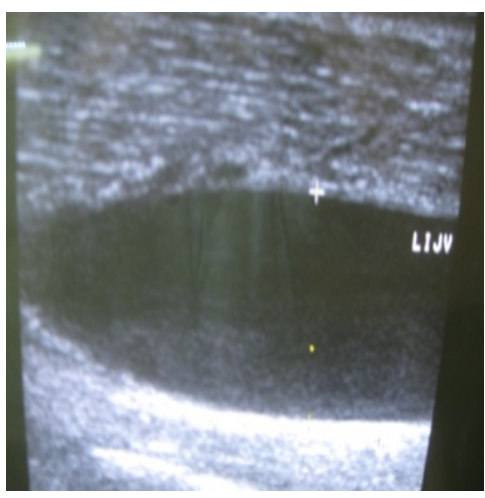

Figure 5.

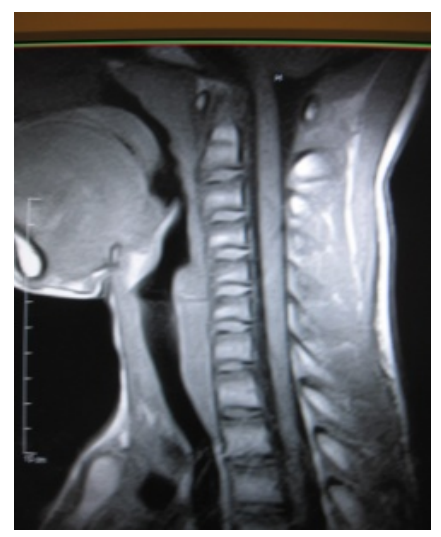

Figure 4.

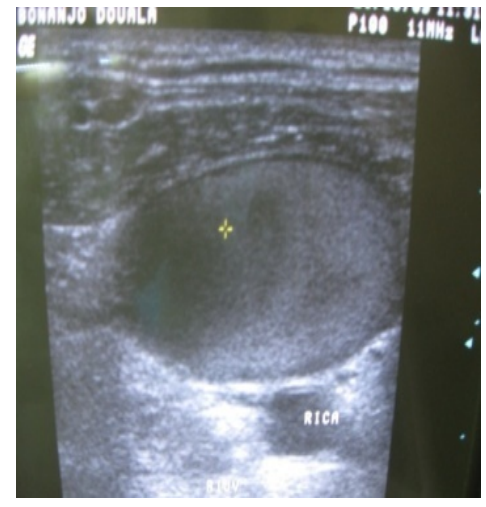

Figure 6.

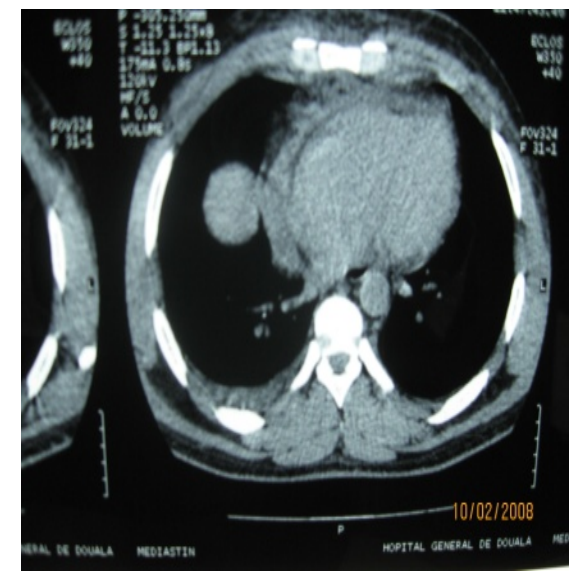

Figure 7. 